\title{
La Banque asiatique de développement et les jeux d'influences chinois et japonais
}

The Asian Development Bank and the Interplay of Chinese and Japanese Influences

\section{Guy Faure}

\section{(2) OpenEdition}

\section{Journals}

Édition électronique

URL : http://journals.openedition.org/transcontinentales/633

DOI : $10.4000 /$ transcontinentales. 633

ISBN : 978-2-7351-1561-7

ISSN : $1775-397 X$

Éditeur

Editions de la maison des sciences de l'homme

\section{Édition imprimée}

Date de publication : 30 juin 2008

Pagination : 107-124

ISBN : 978-2-200-92511-6

ISSN : 1950-1684

\section{Référence électronique}

Guy Faure, «La Banque asiatique de développement et les jeux d'influences chinois et japonais », Transcontinentales [En ligne], 6 | 2008, document 8, mis en ligne le 06 avril 2011, consulté le 08 septembre 2020. URL : http://journals.openedition.org/transcontinentales/633 ; DOI : https://doi.org/ $10.4000 /$ transcontinentales.633 


\title{
La Banque asiatique de développement et les jeux d'influences chinois et japonais
}

\author{
Guy Faure
}

\section{Banques régionales, terrains d'influence : le cas de la BAD}

Les institutions financières internationales, y compris les institutions régionales du Sud, ont dans la majorité des cas été présidées, dirigées et dominées par les Occidentaux. La Banque asiatique de développement ${ }^{1}$ (BAD) constitue une exception notable à cette règle. En effet, le Japon y a occupé, dès le début, une place centrale qui ne lui a jamais été sérieusement contestée. Son poids est tel que la banque multilatérale a pu être perçue comme un outil au service de la diplomatie japonaise, permettant à cette dernière de coupler ses actions diplomatiques bilatérales et multilatérales. Le Japon, resté un temps à l'écart de la communauté internationale en matière de participation aux organisations internationales, a progressivement pris de l'assurance. Les fonctionnaires internationaux japonais sont aujourd'hui présents en plus grand nombre dans ces organisations. Ils permettent à leur pays de prendre plus de responsabilités dans de nombreux secteurs comme la santé, les réfugiés, ou encore la culture, où un Japonais est à la tête de l'UNESCO. La participation grandissante du Japon dans ces institutions va de pair avec sa croissance et avec l'augmentation de son poids économique sinon politique. Toutefois, la montée en puissance d'autres acteurs importants en Asie, la Chine et l'Inde au premier chef, pose la question des changements du statu quo au sein de la Banque.

Dans cet article, nous étudierons plus particulièrement le rôle croissant qu'y joue la Chine. Il est de bon ton d'opposer les visions japonaises et chinoises en matière

1 - On trouve pour la Banque deux sigles possibles en français (BAD ou BasD) comme en anglais (ADB ou AsDB) afin de la distinguer de son homologue africaine, la Banque africaine pour le développement. En Asie et dans le Pacifique, où il n'y a aucune confusion possible avec la banque multilatérale africaine, on a pour habitude d'utiliser les sigles BAD ou ADB, employés par la Banque asiatique de développement dans tous ses documents.

Guy Faure : CNRS, directeur de l'Institut de recherche sur l'Asie du Sud-Est contemporaine (IRASEC), Bangkok. 


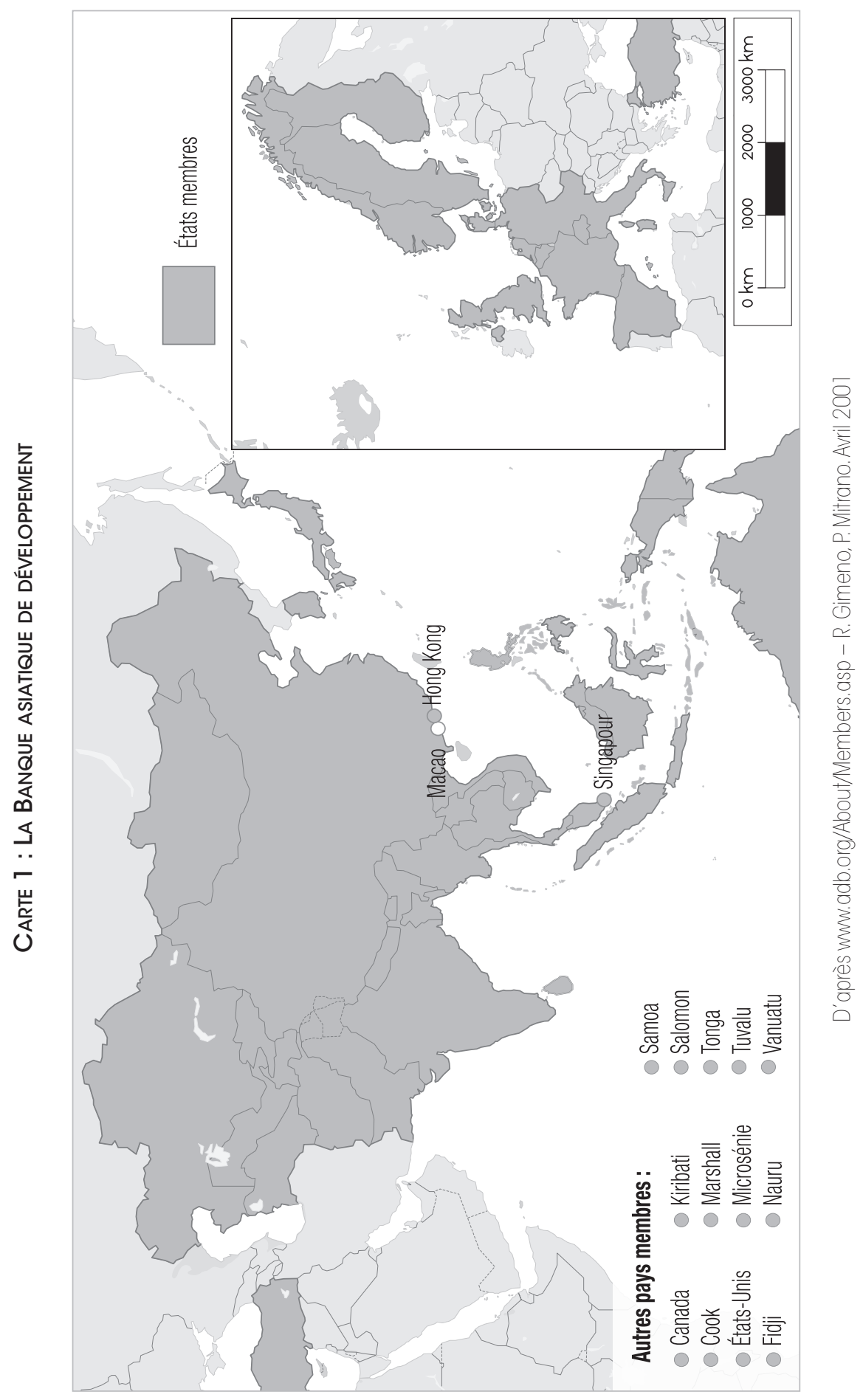




\section{Guy Faure La Banque asiatique de développement}

de géopolitique asiatique et d'évoquer un inévitable jeu de chaises musicales se concluant par un changement de leadership au profit de la Chine. La BAD offre une étude de cas des relations et des interactions entre ces deux pays et les pays de la région; un scénario différent s'esquisse, dans lequel les points de vue auraient plutôt tendance à se rapprocher qu'à diverger.

\section{Le Japon, hégémon bienveillant de la BAD}

\section{Les origines de la banque}

Pour comprendre la place spéciale occupée par le Japon à la Banque asiatique de développement, il faut commencer par rappeler quelques faits au sujet de cette banque multilatérale régionale dont le principe a été adopté en 1963 par une résolution de la Commission économique pour l'Asie et l'Extrême-Orient des Nations unies. Cette commission, basée à Bangkok, sera rebaptisée par la suite Commission économique et sociale pour l'Asie et le Pacifique (CESAP). Les experts de cette instance justifiaient la création de cette banque multilatérale asiatique par la nécessité de répondre à trois besoins ${ }^{2}$ : fournir à la région des ressources financières complémentaires à ses projets d'investissement; aider le financement des projets pas assez dotés par les agences donatrices; assurer le rôle de coordinateur central en matière de coopération économique régionale.

Des considérations secondaires ont également motivé la création de la $\mathrm{BAD}$, notamment la volonté de contribuer à l'émergence d'une expertise locale capable de préparer et de définir les plans appropriés pour le développement de la région, et d'apporter des solutions plus adaptées que celles de la Banque mondiale, perçue à l'époque comme davantage préoccupée par l'aide à l'Inde et au Pakistan et également considérée comme trop occidentale, dans sa gouvernance comme dans l'origine de ses fonds.

À la différence de ses homologues, la Banque de développement africaine (BAfD) et la Banque de développement interaméricaine (BID), la Banque asiatique a ouvert son capital aux pays industrialisés, ses mentors asiatiques considérant à juste titre que l'essentiel des ressources proviendrait de ces pays riches. L'engagement de ces derniers était motivé par la possibilité d'orienter leurs propres politiques de développement tout en préservant leurs intérêts économiques et politiques. Parmi ces puissances industrialisées, le Japon a été conduit tout naturellement à jouer un rôle de premier plan, puisqu'il était la seule nation industrielle en Asie à l'époque. Son expertise et son niveau technologique, mais aussi ses besoins en matières premières, l'expansion de ses marchés d'exportation, ainsi que sa recherche constante de nouvelles opportunités d'investissements, lui ont conféré immédiatement un poids particulier au sein de l'organisation.

2 - Nihal Kappagoda, The Asian Development Bank, The Multilateral Development Banks, vol. 2, Londres, Intermediate Technology Publications, 1995, p. 14. 
Les Japonais ont toutefois su garder un profil très discret au cours de la phase initiale de réflexion sur la création d'une banque multilatérale. C'est malgré tout leur schéma, élaboré avant les négociations de 1963, qui fut adopté en dépit des réticences américaines. Les États-Unis se sont rangés à ce projet à la suite de leur engagement croissant au Vietnam, qui les obligeait à ménager leurs alliés japonais, et parce qu'il fallait apporter des moyens supplémentaires au développement de la région. Une fois le soutien américain acquis, les Japonais ont annoncé leur engagement financier dans le projet. Ces deux décisions ont rendu possible l'établissement en 1966 de la BAD, avec Takeshi Watanabe comme premier président. Depuis, les présidences japonaises se sont succédé sans discontinuité, confirmant le rôle prééminent accordé au Japon pour conduire les destinées de cette organisation financière régionale.

\section{TABLEAU 1 : Les PrÉSIDENTS DE LA BAD (1966-2008)}

\begin{tabular}{|c|c|c|}
\hline & Nom & Période \\
\hline 1 & Takeshi Watanabe & 24 novembre 1966 - 24 novembre 1972 \\
\hline 2 & Shiro Inoue & 25 novembre 1972 - 23 novembre 1976 \\
\hline 3 & Taroichi Yoshida & 24 novembre 1976 - 23 novembre 1981 \\
\hline 4 & Masao Fujioka & 24 novembre 1981 - 23 novembre 1989 \\
\hline 5 & Kimimasa Tarumizu & 24 novembre 1989 - 24 novembre 1993 \\
\hline 6 & Mitsuo Sato & 24 novembre 1993 - 15 janvier 1999 \\
\hline 7 & Tadao Chino & 16 janvier 1999 - 31 janvier 2005 \\
\hline 8 & Haruhiko Kuroda & jer février 2005 à aujourd'hui \\
\hline
\end{tabular}

Source : Tomoyo Saito, Kyoko Ishida, ADB and Japan, NGO Forum on ADB, Manille, mars 2007.

\section{TABLEAU 2 : JAPONAIS OCCUPANT DES POSTES DE DIRECTION À LA BAD}

\begin{tabular}{|c|c|}
\hline Nom & Position \\
\hline Mikio Kashiwagi & Treasurer, Tresor Department \\
\hline Kazu Sakai & Director General, Strategy and Policy Department \\
\hline Kensaku Munenaga & Director General, Budget, Personnel \& Management Systems Department \\
\hline Jo Yamagata & $\begin{array}{l}\text { Director, Infrastructure Finance Division 2, Private Sector Operations } \\
\text { Department }\end{array}$ \\
\hline Katsuji Matsunami & $\begin{array}{l}\text { Director, Agriculture, Environment and Natural Resources, Central \& West } \\
\text { Asia Department }\end{array}$ \\
\hline Kunio Senga & Director General, South Asia Department \\
\hline Kazuhiko Higuchi & Director, Transport and Communications, South Asia Department \\
\hline Yukiko Kojima & $\begin{array}{l}\text { Director, Country Coordination and Regional Cooperation, South Asia } \\
\text { Department }\end{array}$ \\
\hline Tadashi Kondo & Country Director,India Resident Mission, South Asia Department \\
\hline Ayumi Konishi & Country Director,Vietnam Resident Mission, Southeast Asia Department \\
\hline Kuniki Nakamori & Senior Advisor, Southeast Asia Department \\
\hline Toru Shibuichi & Country Director, People's Republic of China, East Asia Department \\
\hline
\end{tabular}




\section{Guy Faure La Banque asiatique de développement}

La BAD justifie son surnom de «banque pour la moitié du monde $e^{3}$ » tant sa zone de compétence géographique est étendue: Asie orientale, Asie méridionale et Asie centrale, ainsi que les îles du Pacifique, dont l'Océanie, soit quarante-huit pays. À ces pays s'ajoutent dix-neuf autres pays d'Europe et d'Amérique du Nord qui contribuent financièrement à son capital. Au total, soixante-sept ${ }^{4}$ pays en sont membres. Parmi les principaux contributeurs de la banque figurent le Japon, les États-Unis, puis la Chine suivie de l'Inde, de l'Australie, et de l'Allemagne. La France se situe au douzième rang.

Le pouvoir dans la banque est fonction du nombre de voix dont dispose chaque pays, lequel est proportionnel aux parts dans le capital. Sur le plan formel, le Japon et les États-Unis sont au même niveau. Mais en raison de contributions additionnelles et substantielles du Japon à d'autres fonds gérés par la Banque, il est de loin le premier contributeur. Les États-Unis ont veillé à ce que le Japon ne puisse détenir de jure la première place en tant que plus important donateur, en l'empêchant de procéder à des augmentations de capital qui modifieraient le classement. Le Japon a cependant contourné cette contrainte en contribuant volontairement à d'autres fonds gérés par la banque. Ainsi il a financé très majoritairement l'Asian Development Fund (ADF), en totalité le Japan Special Fund, le Technical Assistance Special Fund et l'Institut de recherche de la BAD à Tokyo, ou encore l'Asian Currency Crisis Support Facility. Cette situation particulière constitue un fait rare dans l'histoire des organisations internationales, qui mérite d'être souligné : bien que les États-Unis et le Japon affichent officiellement le même pourcentage de voix et le même montant de souscription au capital de la banque, le Japon occupe de facto la première place, grâce à ses contributions auxiliaires.

La présidence est, comme on l'a écrit plus haut, réservée traditionnellement à un Japonais (voir tableau 1 ). On retrouve cette prépondérance sur le plan managérial et opérationnel, avec un contrôle japonais sur de nombreux postes de responsabilités au sein de la banque, dont la direction des ressources humaines (tableau 2). Les Japonais se sentent à la BAD presque comme chez eux. Le personnel japonais de la banque est assuré principalement par des détachements de fonctionnaires du ministère des Finances de Tokyo. Le président est un très haut fonctionnaire, généralement avec rang de vice-ministre avant son affectation à Manille. L'actuel président Haruhiko Kuroda a été conseiller spécial du Premier ministre Koizumi et vice-ministre pour les affaires internationales du ministère des Finances. Les fonctionnaires japonais détachés pour une période de quelques années n'ont pas le sentiment d'être vraiment à l'étranger, mais plutôt en période de mobilité à l'intérieur de leur administration.

Cependant, si le Japon exerce une influence marquée sur la politique de la banque en matière de développement, la réciproque est vraie aussi. On constate que la BAD a été très utile aux Japonais pour élaborer leur propre politique d'aide au développement, qui a été redéfinie au début des années 2000. Ainsi, il est probable que la Banque ait

3 - Dick Wilson, A Bank for Half the World. The Story of The Asian Development Bank 1966-1986, Manille, ADB, 1987.

4 - Le Luxembourg a rejoint la banque en 2006. 
autant servi d'outil d'influence que de terrain d'apprentissage pour l'aide au développement, en particulier pour le couplage de l'aide bilatérale avec l'aide multilatérale.

\section{L'agenda stratégique de la BAD}

Aujourd'hui, la BAD s'est donné comme mission principale d'éradiquer la pauvreté de la région. Son "agenda stratégique» comporte un triple objectif : tout d'abord, créer les conditions d'une "croissance économique durable», excluant par exemple une exploitation non contrôlée des ressources naturelles ou générant de fortes pollutions, condition incontournable pour la Banque; second objectif, un «développement social» qui profite au plus grand nombre; le troisième objectif porte sur "une bonne gouvernance», condition d'une gestion saine et sans corruption permettant une répartition juste des fruits de la croissance.

Pour réaliser son "agenda», l'institution multilatérale suit une triple démarche. En premier lieu, faire reposer son action sur le "développement du secteur privé», que la Banque considère être vertueux par rapport à un secteur public jugé trop souvent inefficace et plus coûteux. Cette attitude très favorable au secteur privé lui a souvent été reprochée. La «coopération régionale» représente le second volet de cette démarche, et constitue un des traits les plus caractéristiques de la philosophie du développement de la BAD. Enfin, la Banque met en avant, au moins sur le plan déclaratif, «la protection de l'environnement». Paradoxalement, la BAD, qui se pose en promoteur de la défense de l'environnement, est très attaquée par les ONG environnementalistes, qui lui reprochent régulièrement de ne pas en faire assez et d'être à l'origine de futures catastrophes écologiques et humaines. Le débat est loin d'être tranché, les ONG exagérant les risques écologiques de certains projets de développement, alors que de son côté la BAD néglige les positions des populations directement affectées par certains programmes.

Parallèlement à ses activités bancaires, la BAD organise quantité de réunions internationales avec les gouvernements de la région, les pays donateurs ou les organismes internationaux (Banque mondiale, Fonds monétaire international, Commission économique et sociale des Nations unies pour l'Asie et le Pacifique (CESAP), autres agences des Nations unies), afin de définir et de mettre en œuvre des politiques appropriées, de créer des institutions, ou d'adopter des règlements au niveau régional, national ou local pour faciliter la réalisation des projets.

La charte de la Banque souligne que «la coopération régionale doit être vue comme inhérente aux opérations de la banque». Cette coopération régionale resterait toutefois un vain mot sans harmonisation des politiques de développement des différents États membres en vue de se mettre en phase avec les initiatives régionales de la Banque. Pour mener à bien sa mission, la BAD pose comme principe essentiel que les processus de réforme interne, en particulier pour les pays en transition, aillent dans le sens des impératifs régionaux de croissance et de développement. À ses yeux, les pays les moins développés d'Asie centrale et d'Asie du Sud-Est sont engagés dans une double transition. La première, avec le passage d'une économie reposant sur une agriculture de subsistance à une économie plus diversifiée. La seconde, avec le passage d'une économie planifiée vers une économie de marché. 


\section{Guy Faure La Banque asiatique de développement}

Les initiatives d'institutions internationales comme la BAD ont un impact considérable sur les pays les moins développés et sur ceux en transition, souvent les mêmes en Asie. Bien qu'elle s'en défende, la BAD ne se cantonne pas à de simples tâches de banquier. Elle joue un rôle éminemment politique, tout en adoptant généralement un profil bas et en faisant office de "conseiller du prince " ${ }^{5}$ en matière de développement. Par son caractère "asiatique» malgré une présence non négligeable de membres occidentaux, la $\mathrm{BAD}$ se distingue des institutions financières internationales que sont la Banque mondiale et le Fonds monétaire international, communément perçus comme les représentants des intérêts occidentaux, sinon exclusivement américains. La BAD constitue une exception, celle de ne pas être sous la «tutelle occidentale» mais sous celle, incontestée, du Japon. Depuis la création de l'institution, les Japonais, en assurant la présidence et en occupant une multitude de postes clés, ont également imprégné la Banque de leur culture, d'une certaine dose de "gestion à la japonaise» où le consensus est de règle. Cependant, la culture d'entreprise de la BAD est composite : d'autres influences s'exercent, en particulier celles des Indiens et des représentants d'autres pays du sous-continent qui occupent un grand nombre des postes de cadres supérieurs et moyens à la Banque, sans oublier les Philippins, qui fournissent, avec $50 \%$ des effectifs, le gros des troupes administratives mais également des cadres bien formés à tous niveaux.

\section{La Chine ou le bon élève du multilatéralisme}

La Chine a longtemps souffert d'un certain déficit en matière de participation aux institutions internationales. Toutefois, on observe une présence plus active de ce pays dans le système international consécutif à sa montée en puissance économique. Depuis son entrée à l'ONU en 1971, la Chine a rejoint plus d'une cinquantaine de grandes institutions, et le nombre de ses participations s'est accéléré à partir du milieu des années 1980. Ainsi en 1986 la Chine a-t-elle rejoint la BAD en entrant dans son capital. Son adhésion à l'Organisation mondiale du commerce (OMC) en 2001 marque une date importante dans ce processus d'internationalisation.

En adhérant à ces institutions, la Chine compte exercer ses droits et également jouer pleinement son rôle. Mais sa position vis-à-vis des organisations internationales a évolué depuis 1949 et trois étapes peuvent être identifiées. La Chine a d'abord marqué une grande défiance, à l'époque maoïste, pour «ces instruments occidentaux de l'impérialisme et de l'hégémonisme». Dans une seconde étape ouverte par son entrée à l'ONU en 1971, la Chine a tenté de changer le système international de l'intérieur en développant sa présence dans les organisations internationales. Enfin dans les années 1980, la Chine a cherché à exploiter les possibilités offertes par sa participation au système international pour la promotion de ses intérêts propres.

Vue d'Asie du Sud-Est, la présence chinoise peut paraître menaçante. Toutefois, il semblerait que la Chine ait su rapidement prendre conscience de la menace à la

5 - Elle se présente volontiers comme un «intermédiaire désintéressé», honest broker dans son vocabulaire. 
fois stratégique, économique et politique, qu'elle représentait pour ses voisins du Sud. En réaction, et pour dissiper ces craintes, elle tente de faire évoluer son image, notamment en changeant le style de sa diplomatie.

Le cadre de la BAD permet de s'interroger sur une nouvelle évolution de la part de la Chine par rapport à cette situation de «multilatéralisme intéressé». Il permet aussi de vérifier ou d'avancer un certain nombre d'hypothèses sur l'avenir des relations sino-japonaises, très souvent réduites au présupposé d'un inévitable conflit d'intérêts qui ne peut déboucher que dans une lutte entre les deux puissances asiatiques pour l'hégémonie régionale. Les organisations internationales comme la BAD ne peuventelles fonctionner qu'avec le leadership d'un «pays directeur» ou pays hégémonique, le Japon dans le cas qui nous intéresse? Depuis son entrée à la BAD en 1986, la Chine a fait du chemin à l'intérieur de cette institution. Jusqu'où ira-t-elle? Dans le cas du développement de la Région du Grand Mékong ${ }^{6}$, programme phare de la banque, les visions chinoises et japonaises diffèrent. Vont-elles se contredire ou se rejoindre? Enfin, les «styles diplomatiques» de la Chine et du Japon, très éloignés, ne se rapprocheront-ils pas à terme dans une approche partagée pour une diplomatie plus constructive, plus consensuelle, et plus «douce», celle d'une soft diplomacy ${ }^{7}$ ?

La Chine entend, elle aussi, depuis quelques années, occuper les devants de la scène asiatique, et son implication de plus en plus forte au sein de la Banque a notablement modifié les rapports entre Pékin et Manille, siège de la BAD. En effet, depuis son adhésion en 1986, la Chine a largement tiré profit de son appartenance à la banque multilatérale en devenant aujourd'hui son deuxième plus grand débiteur, devancée seulement par le Pakistan, membre depuis l'origine et principal bénéficiaire des financements de la BAD et de l'aide technologique. La contribution technologique de la BAD à la Chine a couvert environ 500 projets entre 1986 et 2005. En 2006, la Chine se situait au premier rang des bénéficiaires pour les prêts à l'État, avec près de 1,6 milliard de dollars, près de $25 \%$ du total des montants prêtés, et au second rang pour les prêts au secteur privé après l'Inde. Mais, fait tout aussi remarquable, la Chine est aujourd'hui devenue un contributeur de premier plan à l'institution financière, et figure à la troisième place en tant qu'actionnaire, avec 6,45 \% des parts du capital et 5,46 \% des droits de vote, ce qui la place après le Japon et les États-Unis.

La Chine est également présente à travers ses représentants dans la Banque. Le personnel de la BAD est composé pour moitié d'employés philippins. Les Japonais arrivent ensuite avec 122 représentants qui occupent des postes clés, suivis par les

6 - Rappelons qu'en français le terme « région», proche du terme de "province» (Corse, Bretagne), fait référence à un espace infranational. Nous l'employons ici dans son acception anglaise d'espace supranational. Il faut noter que le terme de "sous-région", qui renvoie dans le vocabulaire anglosaxon à une division sous-continentale du monde, a été adopté par l'administration française dans ses documents concernant "la sous-région du Grand Mékong». Le mot subregion n'ayant pas d'équivalent en français nous choisirons ici de traduire Greater Mekong Subregion par « Région du Grand Mékong».

7 - Allusion au concept de soft power, puissance bienveillante, inventé par Joseph Nye, Soft Power : The Means to Success in World Politics, New York, Public Affairs, 2004, p. 5-6. Le concept est développé pour le cas de la Chine par Joshua Kurlantzick, Charme Offensive, How China Soft Power is Transforming the World, New Haven et Londres, Yale University Press, 2007. 


\section{Guy Faure La Banque asiatique de développement}

Américains (110) et les Indiens (95). Les Chinois occupent le quatrième rang du classement avec un effectif de 87 personnes, en croissance ces dernières années. En sus de jeunes cadres de plus en plus nombreux et de brillants experts, le viceprésident chinois de la banque, Liqun Jin, est assez emblématique de l'importance que place la République populaire dans le choix de ses représentants. Avant de rejoindre la $\mathrm{BAD}$, Liqun Jin a occupé le poste de vice-ministre pour le budget au sein du ministère des Finances de son pays, ce qui le situe à un rang équivalent à celui du président japonais de la BAD, qui a occupé une position similaire dans son pays. Il en va différemment avec les autres vice-présidents, ce qui traduit des enjeux différents : l'Américain est un diplomate de carrière, la vice-présidente allemande est une haute fonctionnaire du ministère de l'Économie de la République fédérale, et le dernier vice-président est un cadre supérieur issu de la BAD.

En mars 2005, la Chine, suivant à une échelle encore modeste les pas du Japon, a créé un fonds de 20 millions de dollars destiné à la réduction de la pauvreté dans les pays asiatiques en voie de développement. En avril de la même année, le président Hu Jintao avançait à Manille une proposition en trois points sur le renforcement de la coopération entre la Chine et la Banque asiatique de développement. Le président chinois a indiqué à Haruko Kuroda, président de la $\mathrm{BAD}$, que son pays accorderait une grande attention à sa coopération avec les organisations internationales, les trois domaines pour lesquels il souhaite un renforcement de l'action de la Banque étant d'abord l'extension des domaines de crédits touchant la construction des infrastructures, l'agriculture, la santé, la culture, l'éducation et la protection de l'environnement; puis, le renforcement de la coopération régionale et sous-régionale, en Asie du Sud-Est et du Nord-Est; et enfin, le renforcement de la coopération dans la lutte contre la pauvreté.

Intervenant au deuxième sommet du Programme de coopération économique du Grand Mékong à Kunming en juillet 2005, Wen Jiabao, le Premier ministre chinois, a rappelé les contributions de son pays, qui participe aux grands projets d'infrastructures de la BAD, comme l'autoroute de Kunming à Bangkok, l'aménagement du cours supérieur du Mékong, ainsi qu'à des programmes de formation dans divers domaines, allant de l'agriculture aux affaires douanières. Il a également mentionné les prêts et les dons importants octroyés par la BAD à son pays. Il a ajouté ce commentaire: "[la BAD...] a favorisé notre coopération sous-régionale et s'est fait un partenaire important des pays du Grand Mékong... nous souhaitons que la BAD joue un rôle plus grand encore dans la coopération future».

Dans le domaine de l'aide au développement, la Chine a changé de camp : de bénéficiaire, elle devient à son tour un pays accordant dons et prêts à des pays en voie de développement d'Asie et d'Afrique. L'autre BAD, la Banque africaine de développement, a tenu son assemblée générale à Shanghai les 16 et 17 mai 2007 sur le thème "Afrique-Asie, partenaires de développement», car la Chine est l'un des 24 membres non africains de l'organisation, au côté de 53 actionnaires africains, venus «s'inspirer de l'expérience de l'Asie en matière de réduction de la pauvreté». En Asie même, la Chine a accordé en octobre 2006 un prêt de 200 millions de dollars au Cambodge (après l'annonce de l'annulation d'un milliard de dollars de dette) pour améliorer les routes et construire des ponts sur les principaux fleuves : un pont sur le Mékong 


\section{Carte 2 : Grand Mékong}

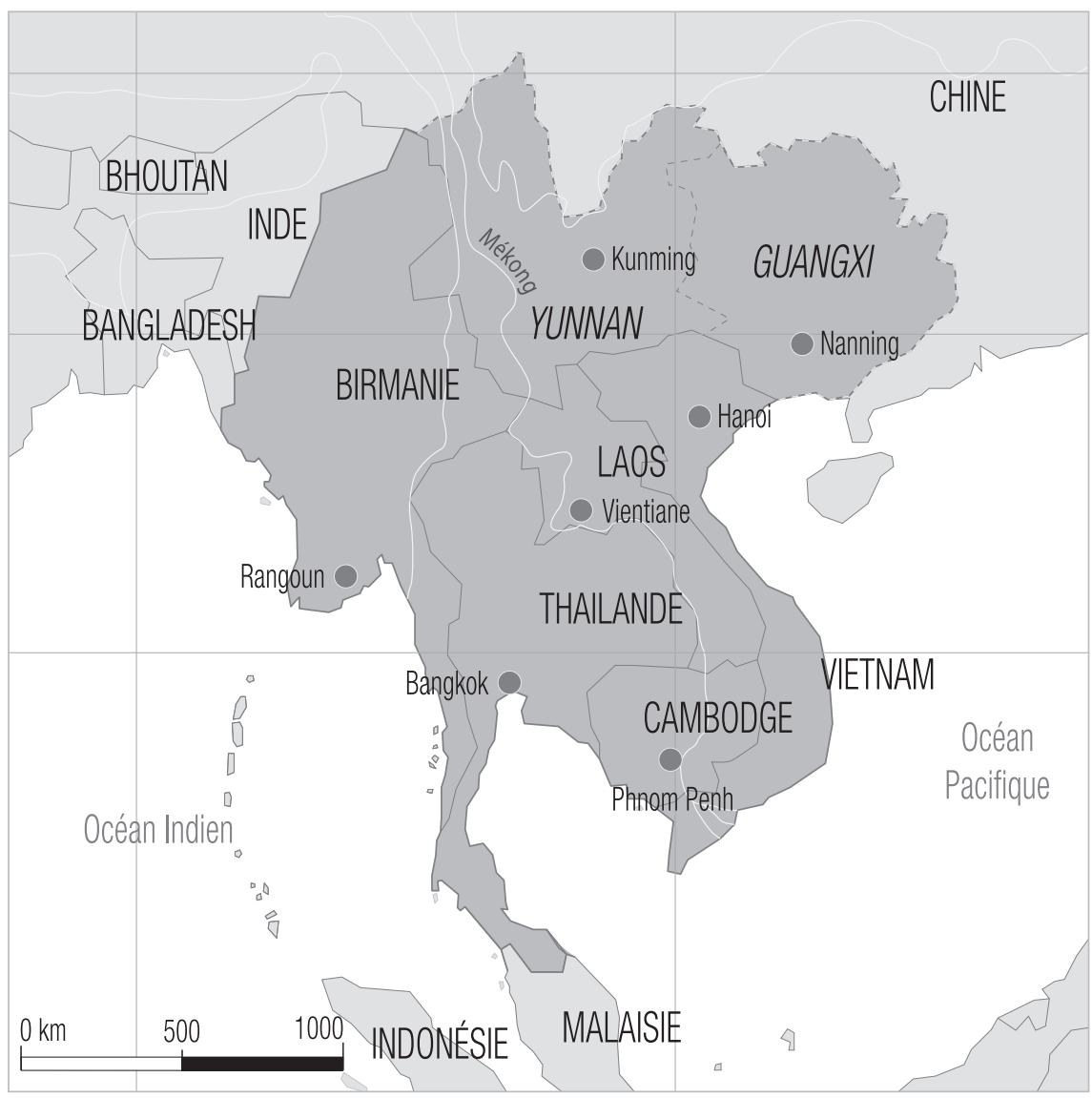

de 1060 mètres et un autre en aval du Tonle Sap de 957 mètres. Dans le domaine du développement de réseaux ferrés, la Chine est également très active. Elle participe au chemin de fer pan-asiatique, dont la première section Chine-Vietnam fera le lien entre Kunming, capitale du Yunnan, et le réseau vietnamien via Hanoi.

Ainsi en moins d'une décennie, la Chine a réussi son insertion dans le système international et dans ses organismes multilatéraux, rejoignant le Japon dans cette voie.

\section{Le nouveau "grand jeu» géopolitique autour du Mékong}

Au début de la décennie 1990, le concept géographique de "région» ou "sousrégion ${ }^{8}$ du Grand Mékong reste encore vague. Mais l'idée de rassembler les pays riverains du fleuve Mékong, tous plus ou moins impliqués dans les guerres

\footnotetext{
8 - Voir note 6 .
} 


\section{Guy Faure La Banque asiatique de développement}

indochinoises, va prendre corps. Très vite cependant se pose la question de la sélection des pays membres de cet espace nouveau. Pas d'objection pour la Thaïlande : de par sa position centrale, elle est indispensable à la réalisation du plan, aucun projet ne pouvant se concevoir sans elle. La proximité géographique et culturelle ${ }^{9}$ de la Chine, qui répond également au critère de pays traversé par le Mékong, a pesé en faveur de son intégration, ainsi que les efforts diplomatiques de personnalités influentes, à l'image de Cesar Virata, ancien Premier ministre philippin (19811986 ) et un des pères fondateurs de l'ASEAN, qui voyait la Chine jouer un rôle crucial pour la région. Si la présence chinoise a été rapidement acquise, de nouvelles négociations se sont tenues pour en déterminer les modalités. L'idée d'inclure une seule province, celle du Yunnan, a finalement été préférée à l'adhésion de la Chine dans son entier, solution défendue par Pékin, ou à l'adhésion de provinces supplémentaires, comme celle du Guangxi. Cette dernière proposition, rejetée initialement, a fini par être acceptée en 2005. Elle constitue assurément une victoire de la diplomatie chinoise, car l'adhésion de cette région côtière chinoise contredit le principe fondateur du projet d'inclure les pays limitrophes du fleuve Mékong.

Cette coopération régionale s'est inspirée pour son mode opératoire de deux contre-exemples : la Commission du Mékong (Mekong River Commission ou MRC) ${ }^{10}$ et l'ASEAN. Si l'Association des nations de l'Asie du Sud-Est a eu le grand mérite de contribuer à la stabilité politique de la région, son bilan est plus contesté en matière de coopération économique. Traditionnellement, les négociations étaient menées au niveau des ministères des Affaires étrangères des pays membres, ce qui a souvent conduit à des discussions stériles au cours desquelles les différents interlocuteurs ont toujours privilégié une défense étroitement chauvine de leurs intérêts nationaux au détriment de l'intérêt régional. La Commission du Mékong a apporté des enseignements complémentaires, sur ce qu'il fallait éviter. L'expérience de la Commission a d'une part confirmé la difficulté qu'il y a à travailler avec les administrations des affaires étrangères dans les accords sur l'eau, cas d'école de la complexité d'une «hydrodiplomatie» régionale ${ }^{11}$. Elle s'est d'autre part fondée sur une charte si contraignante que les Chinois et les Birmans ont refusé d'y adhérer, préférant rester observateurs. Le grand mérite de la Commission est en revanche d'avoir constitué un forum fécond d'idées sur les questions hydrauliques, l'eau du bassin représentant une ressource stratégique pour la région ${ }^{12}$.

Le Japon maîtrise bien la complémentarité diplomatique des niveaux multilatéral et bilatéral, ce qui lui permet de rester très actif dans la région. Il a initié des réunions

9 - Du fait d'une importante présence de Chinois de la diaspora très intégrée dans toutes les strates de la société thaïlandaise.

10 - Christian Taillard, "Le Laos à la croisée des corridors de la Région du Grand Mékong», in D. Gentil et Ph. Boumard (dir.), Le Laos doux et amer, Paris, CCL-Karthala éditions, 2005, p. 71-73.

11 - Voir sur ce thème l'étude de Bastien Affelstranger, Le Mékong, fleuve et territoires. Hydropolitique en Asie du Sud-Est, IRASEC, à paraître en 2008.

12 - La thèse de Luc Lacroze, Le Mékong du Yunnan à la mer de Chine, contribution à l'étude de l'aménagement d'un fleuve tropical, Paris, 1991, dresse un bilan sans complaisance de la Commission du Mékong. Elle est parue sous le titre L'aménagement du Mékong 1957-1997. L'échec d'une grande ambition? Paris, L'Harmattan, 1998. 
régulières des ministères économiques avec les pays de la péninsule, Cambodge, Laos, Myanmar, et Vietnam (CLMV). Ces réunions, suivies par les milieux d'affaires japonais, offrent des opportunités d'investissement intéressantes. Mais ces initiatives japonaises sont également motivées par la nécessité de faire contre-feu à la montée rapide de l'influence économique de la Chine dans cette partie du monde. Le Japon qui semblait s'être endormi sur ses lauriers - en 2001, il fournissait $60 \%$ de l'aide à l'Asie du Sud-Est -, a dû se réveiller en sursaut. Il commence à regagner une partie du terrain perdu au bénéfice de Pékin en matière d'influence dans la région. Au début des années 2000, la Chine a adopté son dixième plan quinquennal qui a démarré l'année suivante. Ce plan prévoyait, entre autres mesures, une stratégie de développement de ses provinces «occidentales" plus pauvres afin de réduire l'écart considérable existant avec les provinces côtières économiquement plus avancées. Dans cette nouvelle stratégie, le développement de la région du Mékong fait figure de priorité pour la Chine, qui a pris de nombreuses dispositions pour améliorer ses relations avec ses voisins du Sud. Depuis 2002, elle a réduit ou supprimé les droits de douane pour le Laos, le Cambodge et la Birmanie, sur plus de 600 produits. Avec les cinq autres membres de la Région du Grand Mékong, elle a multiplié les accords dans les domaines des transports, de la santé, du commerce, des communications et de la protection de l'environnement. Du reste, dans la région du Mékong, la géographie joue clairement en faveur de la Chine et non du Japon. Le Premier ministre Wen aime à le rappeler dans ses discours, comme il le fit lors du sommet de Kunming : «Un voisin proche sera d'une plus grande aide qu'un parent éloigné», en ajoutant «je suis convaincu que le développement économique de la Chine profitera non seulement à sa population de plus d'un milliard d'hommes, mais également offrira des possibilités de développement pour d'autres pays, ses voisins en particulier, et contribuera ainsi à la stabilité et la prospérité de la région et du monde ${ }^{13}$.

La participation chinoise dans ce programme régional a très certainement contribué à un retournement de la stratégie régionale, comme le constate Christian Taillard : «Ces partenariats [ASEAN+3, et accord de libre-échange ASEAN-Chine pour 2010] renforcent la coopération à l'échelle de la région du Grand Mékong... ils permettent de tester la nouvelle stratégie régionale chinoise, en rupture avec l'ancienne politique étrangère limitée aux seules relations bilatérales ${ }^{14}{ }$.

\section{Chine Japon : vers une convergence diplomatique?}

Aujourd'hui, l'émergence économique chinoise, par son ampleur et son rythme, peut apparaître comme une menace, mais il ne faut pas oublier trop vite qu'il y a quelques décennies, c'est le Japon qui tenait cette place de puissance économique agressive et prédatrice. Grâce à sa diplomatie, le Japon a su rassurer ses partenaires sur ses intentions qui n'étaient pas de conquérir le monde et, de fait, sa croissance économique a profité à l'ensemble de la région. Le développement des relations de la Chine avec les pays de l'ASEAN est parti d'une base très modeste par comparaison avec les autres

13 - «La Chine prend part à la coopération de la Région du Grand Mékong de façon dynamique et pragmatique», www.China.org.cn du 5 juillet 2005.

14 - Christian Taillard, art. cit., p. 71-73. 


\section{Guy Faure La Banque asiatique de développement}

partenaires économiques de cette région. Les progrès rapides et visibles de la Chine n'ont pas pour l'instant bouleversé les relations des autres fournisseurs ou clients des pays de l'ASEAN, dont certains comme les Japonais et les Européens, ont conforté leur présence et leurs échanges avec la zone dans le même temps.

Le raz-de-marée chinois annoncé par la presse est donc à relativiser. Les investissements chinois, bien qu'en progression, représentent moins de 1,5 milliard de dollars, dont la moitié investie à Singapour, soit environ $8 \%$ des investissements directs à l'étranger (IDE) chinois dans le monde, contre 40 milliards d'investissements de l'ASEAN en Chine. La part chinoise des IDE en Asie du Sud-Est est presque imperceptible, avec $0,3 \%$ du total, en regard des parts respectives des Européens (28,8\%), des Américains (17\%), et des Japonais (13\%). Sur le plan de l'aide au développement, l'assistance au Cambodge, au Laos et à la Birmanie reste encore faible par rapport au niveau des contributions japonaises qui représentent 60 \% de l'aide à la région, devançant de loin la Banque mondiale. En la matière, la Chine consacre plus du tiers de son aide à la seule Corée du Nord et elle apporte des moyens importants au continent africain.

Ce n'est donc ni dans les investissements ni dans l'aide au développement que les changements sont les plus perceptibles, mais dans le commerce chinois avec l'Asie du Sud-Est, encore qu'il ne faille pas non plus exagérer, pour l'instant, l'ampleur du phénomène. On constate une accélération de la croissance des échanges commerciaux, de 20 \% par an dans les années 1990 à $30 \%$ au début de la décennie suivante, pour atteindre rapidement et dépasser en 2004 les 40 \% d'augmentation, ce qui représente un montant d'échanges d'environ 109 milliards de dollars. Des chiffres qu'il convient cependant de modérer en rappelant que les entreprises étrangères en Chine constituent une part déterminante des exportateurs et des importateurs (62\% des 500 premiers importateurs et $77 \%$ des 200 premiers exportateurs du pays). Dans le cas de l'ASEAN, les entreprises étrangères composaient 60,6 \% des échanges avec la Chine en 2005. Enfin, les entreprises chinoises ne constituent pas le rouleau compresseur souvent annoncé. La plupart sont de taille moyenne ou petite et ont du mal à concurrencer les entreprises étrangères déjà sur place. De plus, elles ne disposent pas des connaissances et des réseaux sur les marchés de l'ASEAN. Enfin une grande part du commerce chinois provient du Yunnan et du Guangxi qui sont les régions chinoises les plus actives en Asie du Sud-Est, alors que les entreprises chinoises des régions côtières, beaucoup plus puissantes et organisées, concentrent leurs efforts commerciaux vers d'autres marchés, d'Asie du Nord-Est, de Corée et du Japon ou encore d'Amérique du Nord et d'Europe. Les entreprises chinoises sont en concurrence plus directe que les entreprises japonaises ou occidentales avec leurs homologues d'Asie du Sud-Est, car les produits qu'elles exportent sont bien plus souvent similaires à ceux fabriqués dans la région que complémentaires. Autre crainte, bien réelle celle-ci : la croissance des échanges va alimenter l'immigration chinoise dans la péninsule. Ce phénomène est très visible dans le nord du Laos, au Cambodge, et surtout en Birmanie et dans une moindre mesure en Thaïlande. Au total, la croissance globale des échanges commerciaux de la Chine a progressé dans les mêmes proportions que les échanges commerciaux avec sa région voisine du Sud, le facteur important étant bien entendu, du point 
de vue de Pékin, le désenclavement des provinces occidentales de la Chine. Quant aux effets anticipés des accords de libre-échange entre la Chine et le Japon, ils n'ont encore contrarié en rien les rapports avec les autres partenaires de l'ASEAN ${ }^{15}$.

Ce rappel montre que la "menace chinoise» est plus fantasmée qu'effective ${ }^{16}$, mais elle a été perçue comme bien réelle par les pays de l'ASEAN et leurs partenaires, à commencer par les Japonais. Il est difficile de dire si les Chinois, généreux, rassurants et consensuels, ont révisé leur approche diplomatique dans la région et leur attitude dans les organisations internationales comme la BAD du fait des réactions de leurs voisins, ou s'ils se sont ajustés à ce nouvel environnement en suivant une "courbe d'apprentissage» plutôt rapide qui les a conduits vers une approche plus «douce» dans leurs négociations. Tactique ou raison? C'est une question qu'on doit poser aux spécialistes des relations internationales de la Chine.

La Chine se positionne dans cette région dans le registre du soft power, et ne se risque guère dans un registre plus sensible des systèmes sociaux et politiques. En l'occurrence, il semblerait que dans la région le développement économique ait remplacé la confrontation idéologique. Le partage d'une certaine vision du développement, qui met entre parenthèses les questions de démocratie ou de droits de l'homme, s'avère un commun dénominateur dans la zone. En tant que bloc sous-régional, la Région du Grand Mékong a montré encore moins d'inclinaison que l'ASEAN à utiliser des sanctions économiques et politiques pour pousser la Birmanie vers des réformes démocratiques. Les deux principaux investisseurs étrangers en Birmanie sont d'ailleurs la Chine et la Thaïlande, devant Singapour et le Japon. Cette situation illustre l'ambiguïté de la position de la $\mathrm{BAD}^{17}$, qui a fini par prendre des mesures conservatrices vis-à-vis de la Birmanie, pourtant membre de son organisation, en suspendant les prêts à ce pays, et en compromettant ainsi la réalisation de sa stratégie de développement des infrastructures (le corridor économique EstOuest par exemple) au sein de la Région du Grand Mékong. Plusieurs membres de la Banque asiatique de développement, dont la Chine, la Thaïlande et l'Inde, ne l'ont pas suivie et prennent à leur charge les financements nécessaires à la mise en œuvre du programme en Birmanie. La question des barrages financés et construits par la Chine sur le haut Mékong et sur d'autres affluents pose un défi à la BAD qui - en raison des critiques sévères d'ONG et de mouvements de citoyens - avait mis en place pour les barrages qu'elle cofinance dans la région de sévères cahiers des charges en matière d'environnement et de protection des populations, en particulier des minorités ethniques concernées. La Chine dispose d'un savoir-faire nouveau, dans la mesure où près de la moitié des grands barrages de la planète se trouvent sur son territoire. Elle dispose aussi de moyens financiers considérables qui lui permettent de s'affranchir de partenaires étrangers et de leurs exigences techniques ainsi que de

15 - Sheng Lijun, «China in Southeast Asia : The limit of Power», Japan Focus, 4 août 2006, www. japanfocus.org/products/topdf/2184.

16 - Voir J. Ravenhill, "Is China an Economic Threat to Southeast Asia?» Asian Survey, ${ }^{\circ} 5$, septembre-octobre 2006, p. 253-675.

17 - R. Scortino, «What to do with Burma?» Imaging our Mekong, 5 juin 2007 (http://www.newsmekong.org/what_to_do_with_burma). 


\section{Guy Faure La Banque asiatique de développement}

leurs préoccupations sociales. Reste une autre question sensible, celle de la question de la gestion partagée de l'eau dans le bassin du Mékong.

\section{La question du fonds monétaire asiatique}

Au même titre que la Région du Grand Mékong, la question d'un fonds monétaire asiatique, proposé par le Japon comme remède aux crises financières et monétaires, revient régulièrement sur la table de négociation. L'hypothèse d'un tel fonds a fait l'objet d'un rejet de la part des Américains et, plus surprenant, d'une absence de soutien plus que d'un rejet de la part de Pékin. La BAD serait bien placée pour porter cette initiative. En 2005, une décision a été prise pour que la Banque renforce le système de surveillance de la situation économique des pays de l'ASEAN $+3^{18}$, ce qui lui permettrait d'identifier les dérapages, d'alerter et d'agir pour les prévenir. Par ailleurs, la création d'un bureau d'intégration économique dans le cadre de la Banque est perçue comme l'esquisse d'un secrétariat pour un futur fonds monétaire asiatique $^{19}$. Compte tenu du fait que la Chine et le Japon ont sur le plan financier plus de points communs - des réserves importantes et une monnaie sous-évaluée -, que de points de discordance, on peut anticiper des progrès dans la direction d'un fonds asiatique, même si cette idée soulève de nombreuses objections de la part des Occidentaux, notamment des Américains qui ne sont pas des partenaires silencieux.

Le représentant américain à la $\mathrm{BAD}$ est généralement très actif, sinon critique, dans le cadre des réunions des gouverneurs de la Banque, et celle-ci n'est pas toujours bien perçue par Washington, qui, à la suite de la crise asiatique de 1997, a proposé de fusionner la BAD et la Banque mondiale, considérant qu'il y avait double emploi en la matière. Cette question continue de soulever de virulentes controverses. L'argumentaire américain contre l'idée d'un fonds monétaire asiatique se résume à deux points essentiels : cette idée fait courir un "aléa moral ${ }^{20}$ " (moral hazard) et relève du double emploi avec le Fonds monétaire international ${ }^{21}$. Pour Washington, un fonds monétaire asiatique pourrait encourager les pays asiatiques à repousser à plus tard les réformes jugées nécessaires. Pour Eisuke Sakakibara ${ }^{22}$, à l'époque vice-ministre des Finances, la réaction des Américains tenait à ce qu'ils voyaient dans l'idée d'un fonds monétaire asiatique un défi japonais à l'hégémonie américaine en Asie : l'idée de ce fonds était née au ministère des Finances japonais, et cette initiative avait été formulée et soutenue par Haruhiko Kuroda, à l'époque directeur du bureau international du ministère, devenu plus tard vice-ministre des Affaires internationales et huitième président de la BAD en 2005. Il est probable que le dossier du FMA revienne à l'ordre du jour, ce qui pourrait poser de gros

18 - L'ASEAN+3 comprend les dix pays membres de l'ASEAN, plus la Chine, le Japon et la Corée du Sud. (NDLR)

19 - P. Pons, «L'idée d'un Fonds monétaire asiatique est relancée avec l'accord de la Chine et du Japon", Le Monde, 18 mai 2005.

20 - Le concept d'«aléa moral » (moral hazard) se réfère chez les économistes à la théorie de l'information imparfaite, ou aux risques liés à l'asymétrie de l'information.

21 - P. Lipsey, "Japan's Asian Monetary Fund Proposal», Stanford Journal of East Asian Affairs, vol. 3, $\mathrm{n}^{\circ}$ 1, printemps 2003, p. 93-104.

22 - P. Lipsey, art. cit., 2003, p. 99. 
problèmes au sein de la Banque. De fait, la forte présence américaine constitue plus un obstacle aux options proposées par les Japonais que les positions avancées par Pékin. En effet, alors qu'il existe un consensus évident parmi les membres asiatiques de la BAD pour que la Banque adopte une position plus souple vis-à-vis des gouvernements asiatiques, et puisse servir de contrepoids, sinon d'alternative à la Banque mondiale et au $\mathrm{FMI}^{23}$, les Américains s'opposent à cette idée.

En gonflant leurs emprunts à la BAD, la Chine (1,6 milliard de dollars en 2006) et l'Inde (2,5 milliards en 2006) sont devenues, du fait des volumes de leurs remboursements effectués sans délais, les principaux bailleurs de fonds de l'institution alors qu'elles ne détiennent toujours qu'une part modeste du capital. Mais il faudra recapitaliser la Banque à court ou moyen terme. Les pays émergents pourraient alors exiger une part plus importante, ce qui compte tenu des réserves colossales accumulées par la Chine (1 200 milliards de dollars) ne lui poserait aucun problème ${ }^{24}$.

\section{La BAD actrice consensuelle de la construction régionale}

À l'heure actuelle, de tous les programmes régionaux de coopération pour le développement, le projet de Région du Grand Mékong sort clairement du lot, car c'est le seul à présenter des avancées concrètes en termes de croissance. Le projet se distingue aussi par une approche très pragmatique, où certains voient la «marque de fabrique» japonaise caractérisée par un leadership soft, discret et consensuel. La première phase s'étant achevée en 2003, le projet de Région du Grand Mékong est engagé dans une nouvelle phase (2004-2014). À terme, il sera probablement appelé à devenir le laboratoire du développement régional de la BAD. La formule, malgré de nombreuses critiques, semble porter ses fruits car la coopération dans la péninsule Indochinoise fonctionne mieux et sur un rythme plus soutenu que les opérations pourtant plus anciennes conduites dans la partie méridionale de l'Asie du Sud-Est. Force est de constater que la BAD a été plus efficace que l'ASEAN, dont les rares initiatives dans le développement de la péninsule ont consisté à endosser les programmes de la Banque. Le décalage est très perceptible entre l'organisation régionale, en perte de vitesse depuis la crise asiatique, et la banque multilatérale très active dans la péninsule. À ceux qui pensaient - ou qui espéraient - que la Banque asiatique de développement agirait de fait comme la «banque de l'ASEAN», la BAD a prouvé qu'elle pouvait surpasser, avec un certain succès, tant sur le plan de la politique de développement que sur le plan de la diplomatie, une organisation régionale aussi établie que l'ASEAN ${ }^{25}$, sans qu'il existe pour autant de rivalité entre les deux organismes, la BAD jouant plutôt le rôle d'un moteur auxiliaire pour l'ASEAN.

Si la philosophie du développement mise en ouvre par la BAD dans cette partie du monde fait débat, il faut reconnaître à la banque multilatérale l'immense mérite

23 - S. Guttal, "The Asian development Bank/A Problem Institution», Focus on Global South, 9 février 2005, www.socioeco.org/fr/documents.

24 - A. Reverchon, «Trois scénarios pour rénover le système financier mondial», Le Monde, 21 mai 2007.

25 - J.A. Kaplan, The Greater Mekong Sub-region and ASEAN. From Backwaters to Headwaters. Phnom Penh, Cambodian Institute for Cooperation and Peace, 2000. 


\section{Guy Faure La Banque asiatique de développement}

d'avoir réussi à réunir concrètement, autour d'un projet commun, les principaux pays de la péninsule Indochinoise ainsi que la Chine.

Faut-il accorder tout le mérite de ces évolutions économiques et diplomatiques à la $\mathrm{BAD}$, ou faut-il voir cette institution comme le paravent masquant les ambitions du Japon? S'il existe une rivalité sino-japonaise pour le leadership au sein de la BAD, celle-ci reste très discrète. D'évidence, les Japonais devront compter dorénavant avec une présence plus active des Chinois au sein de la Banque asiatique de développement, qui ne sera plus leur chasse gardée. Cette évolution marque probablement la fin de l'époque du condominium nippo-américain sur la BAD, qui laissait exercer de facto le pilotage de la banque au partenaire japonais sous la "vigilante attention» de l'allié nord-américain. Reste à savoir si ces nouvelles relations sino-japonaises pourront s'avérer un moteur dynamique pour la région ou un frein.

Au cours de cette dernière décennie, la Chine a considérablement progressé en influence en Asie du Sud-Est dans tous les domaines, dans l'absolu et par rapport aux positions japonaises dans la zone. Quand bien même la Chine devancerait le Japon au terme d'un processus qui pourrait prendre quelques années voire une décennie, le Japon ne sera pas pour autant éliminé du paysage, car il ne s'agit pas là d'un jeu à somme nulle où le vainqueur remporte toute la mise.

Pour conclure, l'avenir de l'intégration régionale de l'Asie de l'Est, et probablement un jour de l'ensemble Asie orientale et méridionale, se jouera probablement dans la péninsule Indochinoise. Ironie de l'histoire, le montage ASEAN+3 qui servit de point de départ à la construction régionale pour une communauté de l'Asie de l'Est repose sur la réussite du plan du Grand Mékong, programme phare de la Banque asiatique de développement. Si la Banque réussissait son pari de reconstruire la péninsule Indochinoise et d'en faire le point de jonction névralgique entre l'Asie du Sud-Est et l'Asie du Nord-Est, voire aussi avec l'Asie du Sud, elle contribuerait, tant sur le plan strict du développement économique que sur le plan géopolitique, à rapprocher les deux (voire les trois) ensembles géographiques. Le succès de cet enjeu régional dépend du soutien actif de ses membres les plus influents, à commencer par le Japon, la Chine et maintenant l'Inde, de plus en plus intéressée par ses voisins de l'Est. Il dépend également de la cohérence entre les visions régionales de chaque acteur asiatique. Un échec comme celui du projet de fonds monétaire asiatique serait le symptôme révélateur d'un désaccord profond entre les principaux acteurs en présence. À ce titre, la Banque asiatique de développement s'avère bien être, parmi d'autres, un des terrains d'observation privilégiés pour comprendre la réalité des relations sino-japonaises par-delà les grandes joutes diplomatiques habituellement commentées. 


\section{Bibliographie}

- Affelstranger B., Le Mékong, fleuve et territoires. Hydropolitique en Asie du Sud-Est, Paris, IRASEC/Les Indes savantes, à paraître en 2008.

- Asian Development Bank, Annual report 2006, Manille, www.adb.org.

- Economy E., "China's Rise in Southeast Asia : Implications for Japan and the United States», The Journal of Contemporary China, août 2005.

- Faure G., «Le Grand Mékong : pierre angulaire ou pierre d'achoppement de la communauté d'Asie de l'Est?» in S. BoIsSEAU DU Rocher et F. Godement, Asie : entre pragmatisme et attentisme (édition 2006-2007), Paris, La Documentation française (Études de la Documentation française), 2006, p. 127-136.

- Faure G., «La Banque asiatique de développement et l'intégration régionale en Asie», Études internationales, vol. $38, \mathrm{n}^{\circ} 2$, juin 2007, Université Laval, Québec, p. 229-249.

- Gaulier G., F. Lemoine et D. Unal-Kesenci, "China's Emergence and the Reorganisation of Trade Flows in Asia», China Economic Review, $\mathrm{n}^{\circ} 18,2007$, p. 209-243.

- Gilson J., «Complex Regional Multilateralism: Strategising Japan's Responses to Southeast Asia», The Pacific Review, vol. 17, $\mathrm{n}^{\circ} 1$, mars 2004, p. 71-94.

- Gor E., Developing The Mekong. Regionalism and Regional Security China-Southeast Asian Relations, International Institute for Strategic Studies, 2006, 70 pages.

- Gourou P., C. Gironde et J. L. Maurer, Le Vietnam à l'aube du XXI esiècle, bilan et perspectives politiques, Paris, Karthala, 2004.

- Guttal S., "The Asian Development Bank/ A Problem Institution», Focus on
Global South, 9 février 2005, www.socioeco. org/fr/documents.

- Kurlantzick J., China's Charm : Implication of Chinese Soft Power, Policy Brief, Carnegie Endowment for International Peace, $\mathrm{n}^{\circ} 47$, juin 2006.

- Lacroze L., L'aménagement du Mékong 1957-1997. L'échec d'une grande ambition? Paris, L'Harmattan, 1998.

- LincolnE. J., «TheAsian Development Bank Time to Wind it Up?» in M. BENSON (éd.), Reconfiguring East Asia Regional Institutions and Organisations after the Crisis, Londres, Routledge Curzon, 2002, p. 205-255.

- MASAKi H., «China, Japan tug-of-war over Indochina», Asia Times, 5 octobre 2005.

- Masaki H., «Japan Vies China for Dominance in Indochina and ASEAN », Asia Times, 22 mai 2007.

- Ravenhill J., "Is China an Economic Threat to Southeast Asia?" Asian Survey, $\mathrm{n}^{\circ}$ 5, septembre-octobre 2006, p. 653-674.

- SaIto T. et K. Ishida, ADB and Japan, NGO Forum on ADB, Manille, mars 2007.

- SCORTine R., What to do with Burma? site Web «Imaging Our Mekong», www.newsmekong.org/what_to_do_with_burma.

- Taillard C. et D. Kermel Torres, «La région du Grand Mékong, un espace transnational péninsulaire en Asie du Sud-Est continentale», in C. TAILlARD (dir.), Intégrations régionales en Asie Orientale, Paris, Les Indes savantes, 2004.

- Taillard C., «Le Laos à la croisée des corridors de la Région du Grand Mékong", in D. Gentil et P. Boumard (dir.), Le Laos doux et amer, Paris, Comité de coopération avec le Laos-Karthala éditions, 2005. 